\title{
Impact of the Great East Japan Earthquake and Fukushima Nuclear Power Plant Accident on Assisted Reproductive Technology in Fukushima Prefecture: The Fukushima Health Management Survey
}

\author{
Masako Hayashia, b, e, Keiya Fujimoria, c, Seiji Yasumura ${ }^{\text {a, d }}$, Akihito Nakai ${ }^{\mathrm{a}, \mathrm{b}}$, \\ Pregnancy and Birth Survey Group of the Fukushima Health Management Survey
}

\begin{abstract}
Background: The aim of the study was to evaluate the incidences and obstetric outcomes of women who conceived using assisted reproductive technology (ART) procedures in Fukushima Prefecture before and after the Great East Japan Earthquake and Fukushima nuclear power plant accident.
\end{abstract}

Methods: Information was collected and analyzed from 12,070 women who conceived with or without ART in Fukushima Prefecture during the 9 months before and after the disaster.

Results: During the 9 months before and after the disaster, $138(2.0 \%)$ and $102(1.9 \%)$ women conceived with in vitro fertilization-embryo transfer (IVF-ET), respectively. The proportion of women who conceived with IVF-ET decreased during the 2 months immediately after the disaster, but returned to pre-disaster levels 3 months after the disaster. In the case of women who conceived without IVF-ET, the incidences of preterm birth and low birth weight increased after the disaster. In contrast, women who conceived with IVF-ET did not differ significantly in obstetric outcomes before and after the disaster but had a higher incidence of cesarean section and low birth weight compared to those conceived without IVF-ET, regardless of the study period.

Conclusion: The influence of the disaster on woman who conceived using ART procedures was minimal.

Manuscript submitted June 13, 2017, accepted June 26, 2017

${ }^{a}$ Radiation Medical Science Center for the Fukushima Health Management Survey, Fukushima, Japan

bDepartment of Obstetrics and Gynecology, Nippon Medical School, Tama Nagayama Hospital, Tokyo, Japan

'Department of Obstetrics and Gynecology, School of Medicine, Fukushima Medical University, Fukushima, Japan

${ }^{\mathrm{d}}$ Department of Public Health, School of Medicine, Fukushima Medical University, Fukushima, Japan

${ }^{e}$ Corresponding Author: Masako Hayashi, Department of Obstetrics and Gynecology, Tama Nagayama Hospital, Nippon Medical School, 1-7-1 Nagayama, Tama-City, Tokyo 206-8512, Japan. Email: hayashi@nms.ac.jp

doi: https://doi.org/10.14740/jocmr3105w
Keywords: Earthquake; Nuclear accident; Assisted reproductive technology; Perinatal outcome; Pregnancy; Survey

\section{Introduction}

On March 11, 2011, the Great East Japan Earthquake (magnitude, 9.0), followed by a large-scale tsunami, more than 16.0 $\mathrm{m}$ high, in northeastern Japan caused considerable human and property loss. The earthquake and tsunami also caused a major nuclear accident at the Fukushima Daiichi Nuclear Power Plant (Tokyo Electric Company), located in east-central Fukushima Prefecture, about $180 \mathrm{~km}$ southwest of the earthquake hypocenter.

Soon after the disaster, the Fukushima prefectural government performed the Fukushima Health Management Survey to assist long-term healthcare management of residents and to investigate the effects of low-dose radiation exposure caused by the accident [1]. The survey also evaluated pregnancy and birth in Fukushima Prefecture during and after the disaster aimed to improve obstetrical and prenatal care [2]. Disasters such as earthquakes [3-7] and nuclear reactor accidents [8-15] may influence various aspects of perinatal health. Our previous study using the Fukushima Health Management Survey demonstrated that the occurrence of adverse obstetric outcomes, such as preterm birth, low birth weight, and medical complications, was higher in women who conceived after the disaster than in those who were pregnant at the time of the disaster [16].

The Japan Society for Reproductive Medicine (JSRM) also took immediate measures to collect information from assisted reproductive technology (ART) clinics in northeastern Japan after the disaster in order to assess the extent of damage to clinics and to promote revisions of emergency plans [17]. The report documented that 36 of 241 (15\%) clinics had some building damage and $12(5 \%)$ clinics lost at least one embryo [17]. These damages may have affected perinatal medicine in the area because the proportion of pregnancies conceived by in vitro fertilization-embryo transfer (IVF-ET) has recently increased in Japan. 


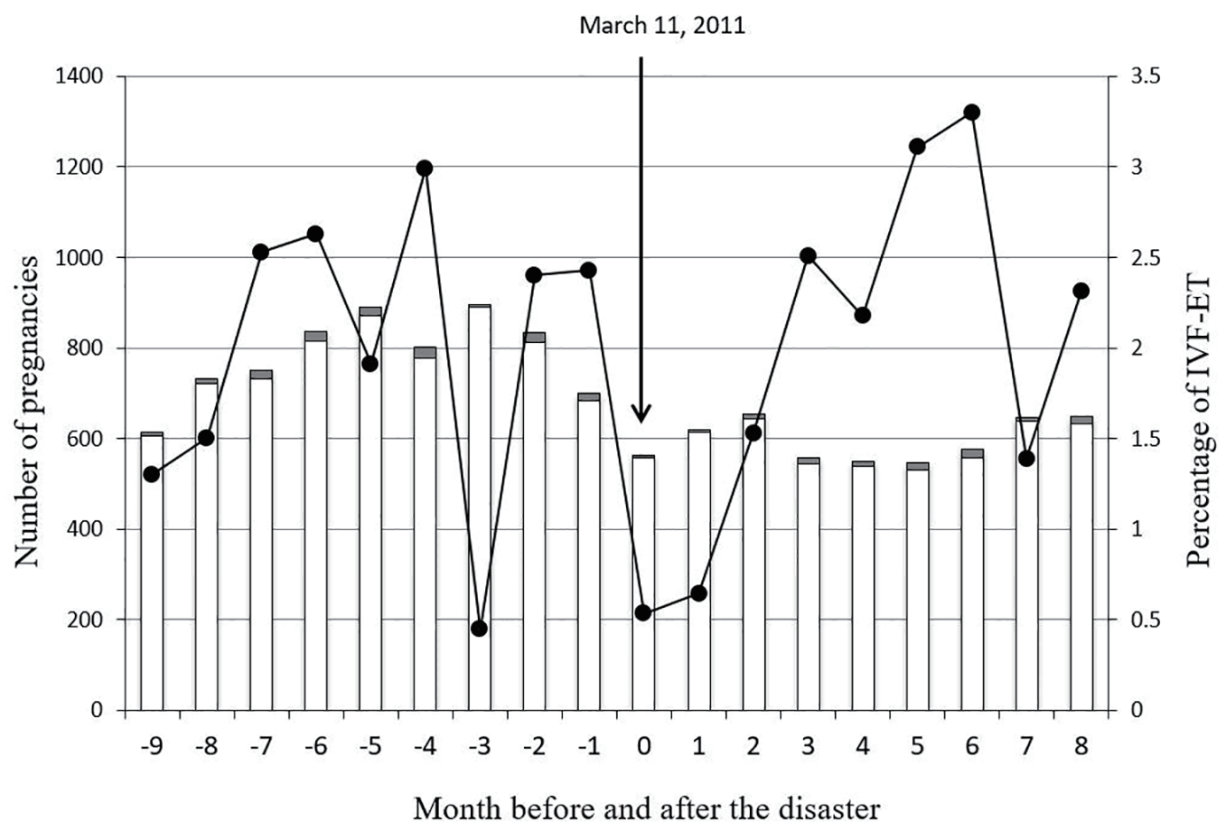

Figure 1. Proportion and trends of women who conceived with in vitro fertilization-embryo transfer (IVF-ET) before and after the disaster. Open and closed columns indicate the number of women who conceived without and with IVF-ET, respectively. Closed circles represent incidences of women who conceived with IVF-ET.

In the present study, we assessed the proportion of women who conceived with ART procedures, and their obstetric outcomes, before and after the disaster in order to evaluate the effect of the Great East Japan Earthquake and Fukushima nuclear power plant accident on ART in Fukushima Prefecture. A detailed pregnancy and birth survey questionnaire included in the Fukushima Health Management Survey performed by the Radiation Medical Science Center (Fukushima, Japan) was used to differentiate and evaluate the perinatal outcomes of pregnancies conceived with ART procedures.

\section{Materials and Methods}

The pregnancy and birth survey was administered to women who received maternal and child health handbooks from municipal officers in Fukushima Prefecture between August 1, 2010 and July 31, 2012, and to women who had handbooks issued in other prefectures, but who received prenatal care or delivered babies in Fukushima Prefecture after the disaster. This survey was approved by the ethics committee of Fukushima Medical University, which is guided by local policy, national law, and the Declaration of Helsinki.

The questionnaires, which can be viewed on the website of the Pregnancy and Birth Survey Group [1], have been mailed since January 18, 2012. A total of 30,517 questionnaires were ultimately distributed. A total of 16,401 responses $(53.7 \%$ response rate) were received as of March 31, 2014.

The questionnaire includes information on maternal and infant characteristics, such as maternal age, parity, history of infertility treatments, medical complications, pregnancy complications, expected date of confinement, gestational age at delivery, birth weight, delivery mode, and major congenital anomalies. These data conform to uniform coding specifications, have passed a rigorous quality check, and have been edited and reviewed by the Radiation Medical Science Center.

Data from 16,126 respondents were included in this analysis after excluding 275 invalid responses. Parity data were also excluded because of the high number of invalid responses, probably due to misunderstanding of the question. To assess the proportion of pregnancies conceived with ART procedures before and after the disaster, the conception date of each pregnancy was estimated from the gestational age at delivery or expected date of confinement. Based on the conception date, data were used from 12,416 women who conceived during the 9 months before and after the disaster (June 11, 2010 to December 10, 2011).

To evaluate the effect of the disaster on the obstetric outcomes of pregnancies conceived with ART procedures, 12,070 women who delivered after March 11, 2011 were included in this analysis. We excluded 113 women who were still pregnant at the time of the questionnaire and 233 women who delivered before March 11, 2011. The remaining participants were categorized into four study groups depending on whether they conceived with IVF-ET and on whether they conceived during the 9 months before (June 11, 2010 to March 10, 2011) or after (March 11, 2011 to December 10, 2011) the disaster. Conception with IVF-ET included women who received eggs spontaneously fertilized by sperm in vitro and eggs fertilized by intracytoplasmic sperm injection, followed by transfer of the embryo back into the uterine cavity. However, further in- 
Table 1. Maternal Characteristics of Respondents

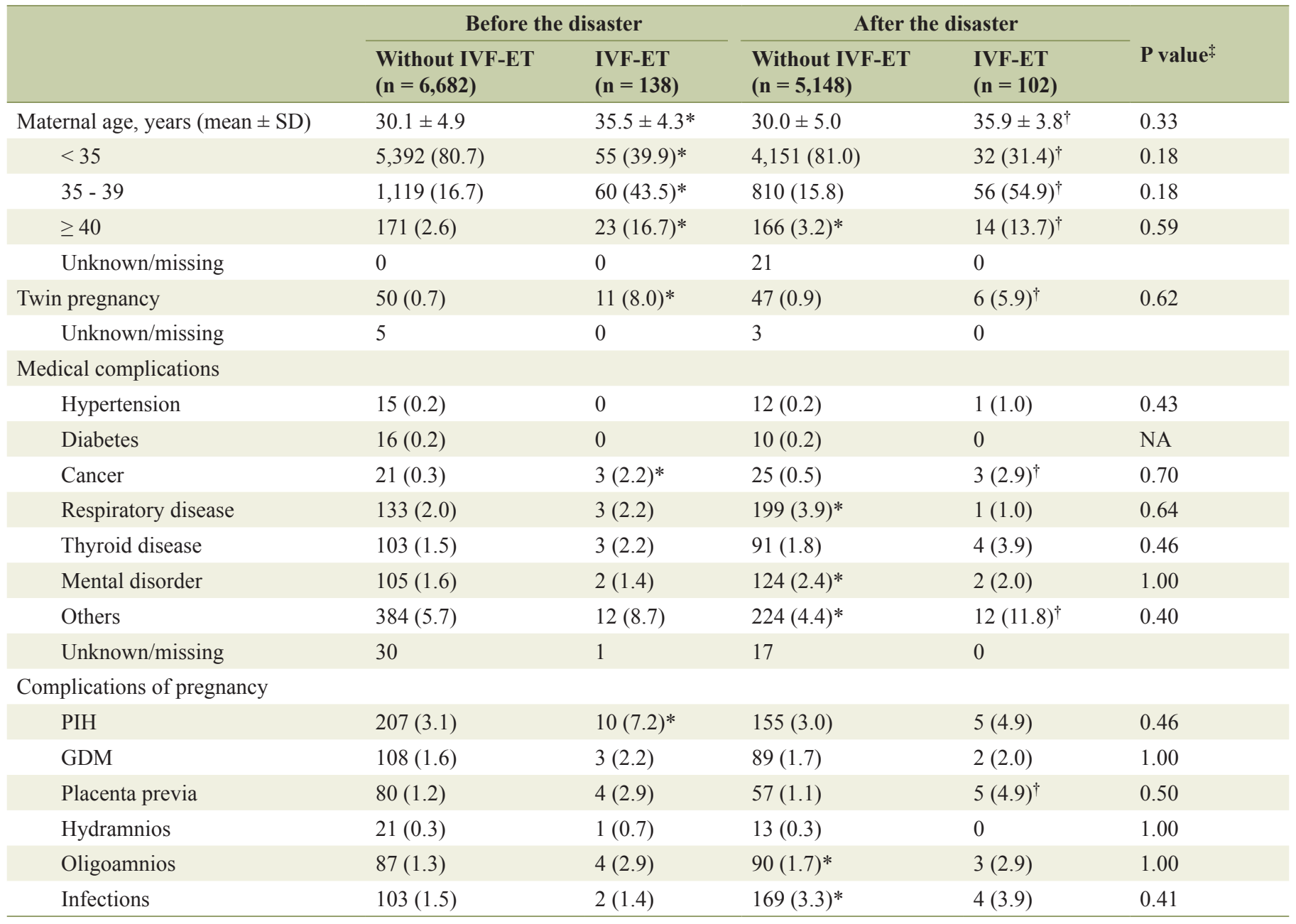

Number in parentheses are the percentage. PIH: pregnancy induced hypertension; GDM: gestational diabetes mellitus; NA: not applicable. ${ }^{*} \mathrm{P}<0.05$, in relation to the without IVF-ET group before the disaster. ${ }^{\dagger} \mathrm{P}<0.05$, in relation to the without IVF-ET group after the disaster. ${ }^{\ddagger}$ Differences between the IVF-ET groups before and after the disaster.

formation on the use of intracytoplasmic sperm injection was unavailable in the questionnaire.

Data were analyzed using SPSS Statistics for Windows, version 17.0 (SPSS Inc., Chicago, IL, USA) and Excel (Microsoft, Redmond, WA, USA). Statistical analysis of continuous variables, such as maternal age, gestational age at delivery, and birth weight, was performed using the Mann-Whitney U test, while categorical variables were analyzed using the Chisquared test. A P value of $<0.05$ was considered statistically significant.

\section{Results}

A total of 6,820 and 5,250 women conceived during the 9 months before and after the disaster, respectively. Of these, $138(2.0 \%)$ and $102(1.9 \%)$ women, respectively, conceived through IVF-ET (Fig. 1). The proportion of women who conceived with IVF-ET decreased dramatically during the 2 months immediately after the disaster. However, the levels returned to pre-disaster levels 3 months after the disaster.

Table 1 summarizes the maternal characteristics of the study groups. There were no significant differences in the characteristics between women who conceived with IVF-ET before and after the disaster. In contrast, among women who conceived without IVF-ET, there was a significant increase in the incidence of maternal complications, such as respiratory diseases, mental disorders, oligoamnios, and infections, in women who conceived after the disaster.

The obstetric outcomes of the study groups are summarized in Table 2. The incidence of cesarean section and low birth weight was increased in women who conceived with IVF-ET, regardless of the study period, compared to women who conceived without IVF-ET.

In women who conceived without IVF-ET, the incidence of preterm birth and low birth weight increased after the disaster. However, among women who conceived with IVF-ET, there were no significant differences in obstetric outcomes be- 
Table 2. Obstetric Outcomes of Respondents

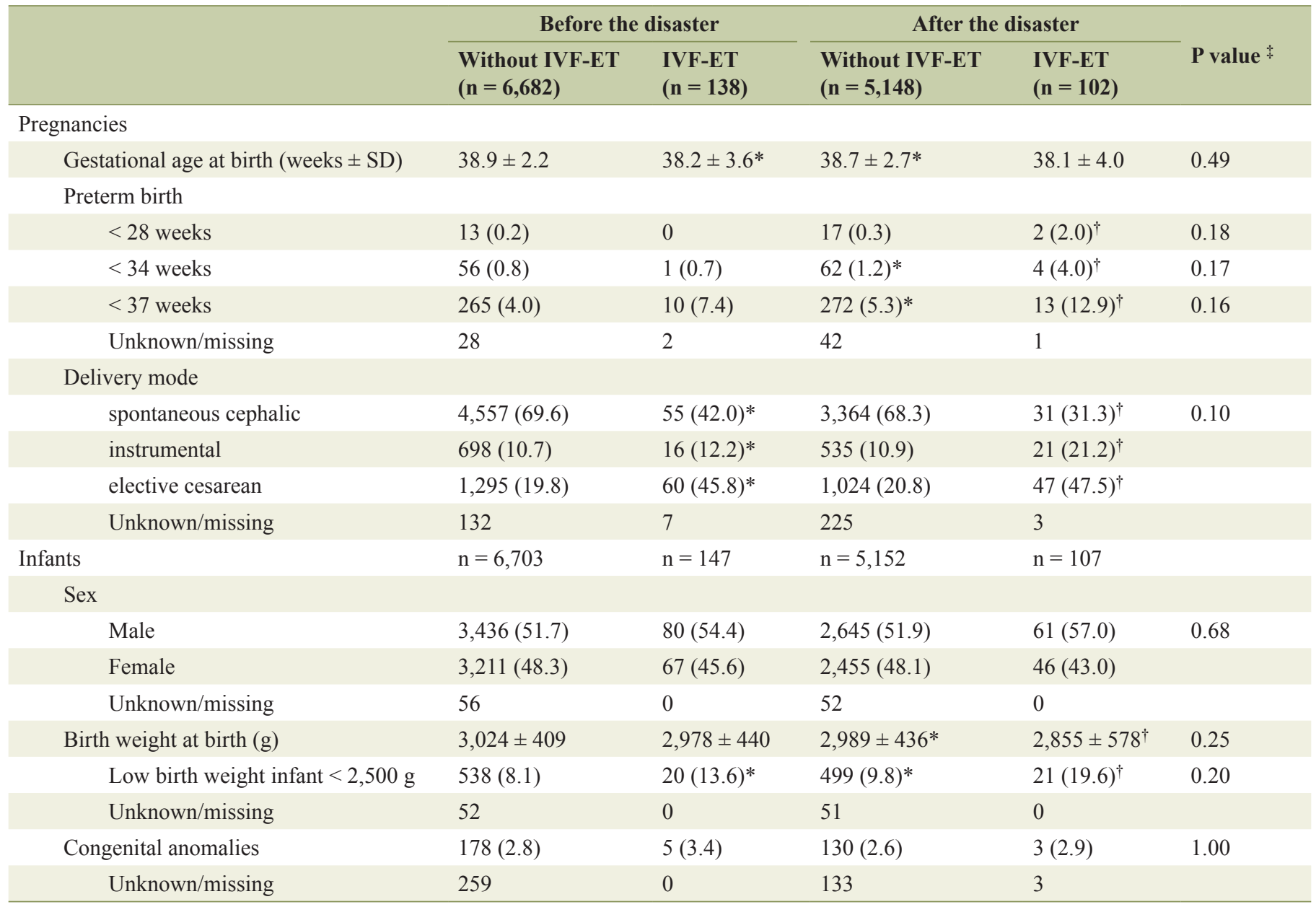

Number in parentheses are the percentage. NA: not applicable. ${ }^{*} \mathrm{P}<0.05$, in relation to the without IVF-ET group before the disaster. ${ }^{\dagger} \mathrm{P}<0.05$, in relation to the without IVF-ET group after the disaster. ${ }^{\circ}$ Differences between the IVF-ET groups before and after the disaster.

fore and after the disaster.

\section{Discussion}

The results of the present study demonstrate that the proportion of women who conceived with IVF-ET in Fukushima Prefecture decreased dramatically during the 2 months immediately after the Great East Japan Earthquake and Fukushima nuclear power plant accident, but returned to pre-disaster levels 3 months after the disaster. Although IVF-ET was associated with increased incidence of cesarean section and low birth weight, there were no significant differences in obstetric outcomes before and after the disaster.

Disasters, such as earthquakes [3-7] and nuclear reactor accidents [8-15], may influence various aspects of perinatal health. However, the influence of such disasters on perinatal outcomes varies according to the scale and area of the event. Many birth complications were reported after the Chinese Wenchuan earthquake in 2008, which caused nearly 70,000 deaths because of its severity [3]. Increased rates of Down syndrome and neural tube defects were reported after the Chernobyl nuclear disaster [9, 11], although data from the most affected areas are limited [8]. In contrast, the Three Mile Island disaster was associated with an increased incidence of depression and anxiety immediately after the disaster [13], but it had no effect on physical functions, such as spontaneous abortion [12].

Despite the damage to the ART clinics in northeastern Japan due to the Great East Japan Earthquake [17], the proportion of women who conceived through IVF-ET in the present study returned to pre-disaster levels 3 months after the disaster, likely because most of the reported damage was minimal, such as cracks in walls and ceilings, dislocation of building joints, and damage to water pipes, although four clinics were partly unusable [17]. In addition, although 12 (5\%) clinics lost at least one embryo, there were no reports of loss of frozen embryos stored in liquid nitrogen tanks. Of the 78 clinics experiencing unexpected power loss, 65 had some form of emergency power backup [17]. These preparations may have reduced the damage.

It is well known that ART procedures are associated with an increased risk of perinatal complications, such as low birth weight, preterm delivery, and cesarean deliveries [18-25]. Preg- 
nancies conceived with ART procedures in this study, regardless of the conception date, too had a higher incidence of adverse obstetric outcomes than non-ART pregnancies. However, there were no differences in obstetric outcomes between the women who conceived with ART procedures before and after the disaster. Taken together, our findings suggest that there was a minimal influence of the disaster on woman who conceived with ART procedures. An unexpected finding in our results was the decrease in the proportion of women who conceived through IVF 3 months before the disaster. This decrease likely reflected the Christmas, year-end, and New Year holidays.

Our previous report, which examined pregnant women living in Fukushima Prefecture, showed that the occurrence of adverse obstetric outcomes was higher in women who conceived within 6 months after the disaster, as compared to those who were pregnant at the time of the disaster [16]. These results suggest that even with no direct exposure to the disaster, the surrounding environment after the disaster might lead to adverse obstetric outcomes. Similarly, there was an increase in adverse obstetric outcomes in non-ART pregnancies after the disaster, as compared to those who conceived before the disaster. In contrast, the influence of the disaster on woman who conceived with ART procedures was minimal. It seems likely that in the women who conceived with ART, their surrounding environment for the pregnancy and delivery has been already managed well 3 months after the disaster. Taking the results of the previous reports and the current study together, the influence of the Great East Japan Earthquake and Fukushima nuclear power plant accident on perinatal outcomes in Fukushima Prefecture was limited as compared to the influence of the Chinese Wenchuan earthquake or the Chernobyl nuclear disaster.

We acknowledge that this study was probably too limited to determine the total influence of the Great East Japan Earthquake and Fukushima nuclear power plant accident because the survey questionnaires included various biases and because the influence of the disaster varied according to the area and passage of time. However, these results demonstrate the disaster had a minimal influence on the proportion and obstetric outcomes of women who conceived with ART procedures. These results, which cannot completely rule out all adverse effects of the disaster, also emphasize the need for continued investigations and careful management of pregnant woman in disaster areas in the future.

\section{Disclosure}

This survey was supported by the National Health Fund for Children and Adults Affected by the Nuclear Incident. The findings and conclusions of this article are solely the responsibility of the authors and do not represent the official views of Fukushima Prefecture government.

\section{Author Note}

The Fukushima Health Management Survey Group Chairpersons: Masafumi Abe (Executive Director, Radiation Medical
Center for the Fukushima Health Management Survey); Shunichi Yamashita, Kenji Kamiya, and Seiji Yasumura (Senior Directors, Radiation Medical Center for the Fukushima Health Management Survey); Makoto Akashi (National Institute of Radiological Sciences); Kazunori Kodama, and Kotaro Ozasa (The Radiation Effects Research Foundation).

\section{References}

1. Yasumura S, Hosoya M, Yamashita S, Kamiya K, Abe M, Akashi M, Kodama K, et al. Study protocol for the Fukushima Health Management Survey. J Epidemiol. 2012;22(5):375-383.

2. Fujimori K, Kyozuka H, Yasuda S, Goto A, Yasumura S, Ota M, Ohtsuru A, et al. Pregnancy and birth survey after the Great East Japan Earthquake and Fukushima Daiichi Nuclear Power Plant accident in Fukushima prefecture. Fukushima J Med Sci. 2014;60(1):75-81.

3. Tan CE, Li HJ, Zhang XG, Zhang H, Han PY, An Q, Ding $\mathrm{WJ}$, et al. The impact of the Wenchuan earthquake on birth outcomes. PLoS One. 2009;4(12):e8200.

4. Chang HL, Chang TC, Lin TY, Kuo SS. Psychiatric morbidity and pregnancy outcome in a disaster area of Taiwan 921 earthquake. Psychiatry Clin Neurosci. 2002;56(2):139-144.

5. Glynn LM, Wadhwa PD, Dunkel-Schetter C, ChiczDemet A, Sandman CA. When stress happens matters: effects of earthquake timing on stress responsivity in pregnancy. Am J Obstet Gynecol. 2001;184(4):637-642.

6. Weissman A, Siegler E, Neiger R, Jakobi P, Zimmer EZ. The influence of increased seismic activity on pregnancy outcome. Eur J Obstet Gynecol Reprod Biol. 1989;31(3):233-236.

7. Harville E, Xiong X, Buekens P. Disasters and perinatal health: a systematic review. Obstet Gynecol Surv. 2010;65(11):713-728.

8. Little J. The Chernobyl accident, congenital anomalies and other reproductive outcomes. Paediatr Perinat Epidemiol. 1993;7(2):121-151.

9. Hoffmann W. Fallout from the Chernobyl nuclear disaster and congenital malformations in Europe. Arch Environ Health. 2001;56(6):478-484.

10. Dolk H, Nichols R. Evaluation of the impact of Chernobyl on the prevalence of congenital anomalies in 16 regions of Europe. EUROCAT Working Group. Int J Epidemiol. 1999;28(5):941-948.

11. Zafra Anta MA, Amor Cabrera MA, Diaz Mier F, Camara Morano C. [Health effects of the Chernobyl disaster. Fifteen years afterwards]. An Esp Pediatr. 2002;56(4):324333.

12. Goldhaber MK, Staub SL, Tokuhata GK. Spontaneous abortions after the Three Mile Island nuclear accident: a life table analysis. Am J Public Health. 1983;73(7):752759.

13. Houts PS, Tokuhata GK, Bratz J, Bartholomew MJ, Sheffer KW. Effect of pregnancy during TMI crisis on mothers' mental health and their child's development. Am J Public Health. 1991;81(3):384-386. 
14. Wertelecki W. Malformations in a chornobyl-impacted region. Pediatrics. 2010;125(4):e836-843.

15. Feshchenko SP, Schroder HC, Muller WE, Lazjuk GI. Congenital malformations among newborns and developmental abnormalities among human embryos in Belarus after Chernobyl accident. Cell Mol Biol (Noisy-le-grand). 2002;48(4):423-426.

16. Hayashi M, Fujimori K, Yasumura S, et al. Obstetric outcomes in women in Fukushima prefecture during and after the Great East Japan Earthquake and Fukushima Nuclear Power Plant Accident: The Fukushima Health Management Survey. Open Journal of Obstetrics and Gynecology. 2016;6(12):705-713.

17. Ishihara O, Yoshimura Y. Damages at Japanese assisted reproductive technology clinics by the Great Eastern Japan Earthquake of 2011. Fertil Steril. 2011;95(8):25682570.

18. Perri T, Chen R, Yoeli R, Merlob P, Orvieto R, Shalev Y, Ben-Rafael Z, et al. Are singleton assisted reproductive technology pregnancies at risk of prematurity? J Assist Reprod Genet. 2001;18(5):245-249.

19. Schieve LA, Meikle SF, Ferre C, Peterson HB, Jeng G, Wilcox LS. Low and very low birth weight in infants con- ceived with use of assisted reproductive technology. N Engl J Med. 2002;346(10):731-737.

20. Retzloff MG, Hornstein MD. Is intracytoplasmic sperm injection safe? Fertil Steril. 2003;80(4):851-859.

21. Zadori J, Kozinszky Z, Orvos H, Katona M, Kaali SG, Pal A. The incidence of major birth defects following in vitro fertilization. J Assist Reprod Genet. 2003;20(3):131-132.

22. Jackson RA, Gibson KA, Wu YW, Croughan MS. Perinatal outcomes in singletons following in vitro fertilization: a meta-analysis. Obstet Gynecol. 2004;103(3):551-563.

23. Reddy UM, Wapner RJ, Rebar RW, Tasca RJ. Infertility, assisted reproductive technology, and adverse pregnancy outcomes: executive summary of a National Institute of Child Health and Human Development workshop. Obstet Gynecol. 2007;109(4):967-977.

24. Hayashi M, Nakai A, Satoh S, Matsuda Y. Adverse obstetric and perinatal outcomes of singleton pregnancies may be related to maternal factors associated with infertility rather than the type of assisted reproductive technology procedure used. Fertil Steril. 2012;98(4):922-928.

25. Hayashi M, Satoh S, Matsuda Y, Nakai A. The effect of single embryo transfer on perinatal outcomes in Japan. Int J Med Sci. 2015;12(1):57-62. 